\title{
Maxillary Anterior Tooth Replaced with Immediate Implant along with Combined Graft Materials: A Case Report
}

\author{
Janani Viswanathan ${ }^{1}$, Jayashree Mohan², Parithimar Kalaignan ${ }^{3}$, Ramesh Raju ${ }^{4}$, Vignesh KK Pandian ${ }^{5}$
}

\begin{abstract}
Aim: This case report illustrates the treatment outcome of immediate implant placement replacing the maxillary central incisor with various graft materials like platelet-rich fibrin (PRF), guided bone regeneration (GBR), and alloplast.

Background: Whenever the anterior tooth replacement, especially in the esthetic zone, the patient's demand for the shortening the treatment time has led to the introduction of the immediate implant.

Case description: A 22-year-old male patient reported to our college with chief complaint of frequent failure of crown in the maxillary right central incisor. Preoperative investigations were made. Atraumatic extraction of tooth was done. Dental implant was placed along with PRF, alloplast, and GBR. Cover screw and sutures were placed. Postoperative radiovisiography (RVG) was taken. The temporary removable denture was provided. After 6 months of the healing period, again intraoral periapical radiograph (IOPA) was taken and second-stage surgery of replacing the cover screw with the healing cap was done and after 10 days, implant-level impression was made and restoration was cemented. Follow-up was done. Conclusion: Immediate implant placement has highly predictable means of tooth replacement and shows success rates. For the long-term success of restoration, attempt was made using various graft materials that had shown a soft tissue and hard tissue regeneration and also they fill the jumping distance between the adjacent bone and implants.

Clinical significance: When compared to the conventional procedure of implant placement, implants placed after extraction show a significant result. This case gives an excellent clinical outcome and esthetic was well appreciated. Hence, the quality of life increases.

Keywords: Alloplast, Cone-beam computer tomography, Guided bone regeneration, Immediate implant, Platelet-rich fibrin.

World Journal of Dentistry (2020): 10.5005/jp-journals-10015-1740
\end{abstract}

\section{BACKGROUND}

High success rates have been reported for single tooth restorations, particularly those replacing anterior teeth. Replacement of missing maxillary anterior teeth is of meticulous important for esthetics, function, and overall appearance of the patient. Implant placement in fresh extraction sockets was first reported by Schulte and Heimke in 1976 and termed as immediate implant. ${ }^{1}$ Various studies have been carried out on the success of immediate implant placement as it has the advantages like soft tissue preservation and esthetics. It is the nearest equivalent replacement to the natural tooth and is therefore a useful addition in the management of patients who have missing teeth because of disease, trauma, or developmental anomalies. Various dental implant systems have come up with predictable long-term results backed by good scientific research and clinical trials.

Muller Devan (1952) stated that preservation of what is remaining should be the first and foremost criterion of any treatment procedure. The goal is to satisfy the patient's desire for replacing missing teeth in an esthetic and functional manner by implant therapy, which provides long-term success. To achieve this goal, there should be a careful assessment of the patient's physical and mental status by the clinician. Proper diagnosis and treatment planning are considered as utmost important for the success of an implant. ${ }^{2}$

\section{Immediate Implant Placement}

Immediate implant was first introduced in 1976; Schulte was the first to place implants into fresh extraction sites in humans. ${ }^{1}$ This method involves the implant placement immediately after the

\begin{abstract}
1,5 Department of Prosthodontics, Crown and Bridge and Implantology, Vinayaka Missions Sankarachariyar Dental College, Vinayaka Missions Research Foundation (Deemed to be University), Ariyanoor, Salem, Tamil Nadu, India

${ }^{2-4}$ Department of Prosthodontics, Vinayaka Missions Sankarachariyar Dental College, Vinayaka Missions Research Foundation (Deemed to be University), Ariyanoor, Salem, Tamil Nadu, India

Corresponding Author: Janani Viswanathan, Department of Prosthodontics, Crown and Bridge and Implantology, Vinayaka Missions Sankarachariyar Dental College, Vinayaka Missions Research Foundation (Deemed to be University), Ariyanoor, Salem, Tamil Nadu, India, Phone: +91 9524235241, e-mail: drvjanani@gmail.com
\end{abstract}

How to cite this article: Viswanathan J, Mohan J, Kalaignan $\mathrm{P}$, et al. Maxillary Anterior Tooth Replaced with Immediate Implant along with Combined Graft Materials: A Case Report. World J Dent 2020;11(3): 231-234.

Source of support: Nil

Conflict of interest: None

tooth extraction, and now, it has become a successful, predictable, and alternative treatment modality. ${ }^{3}$ In the case of an immediate implant, the placement must fulfil several preset clinical conditions.

These include the following: primary stability of the implant, osseointegration, proper prosthetic location, and esthetics. An absolute requirement is that the $3-5-\mathrm{mm}$-wide implant must be inserted into the host bone to gain initial implant stability (Nemcovsky et al. 2002; Juodzbalys 2003). ${ }^{4}$

The condition of the socket substantially affects the predictability of osseointegration, while the biotype of the gingiva

() The Author(s). 2020 Open Access This article is distributed under the terms of the Creative Commons Attribution 4.0 International License (https://creativecommons. org/licenses/by-nc/4.0/), which permits unrestricted use, distribution, and non-commercial reproduction in any medium, provided you give appropriate credit to the original author(s) and the source, provide a link to the Creative Commons license, and indicate if changes were made. The Creative Commons Public Domain Dedication waiver (http://creativecommons.org/publicdomain/zero/1.0/) applies to the data made available in this article, unless otherwise stated. 

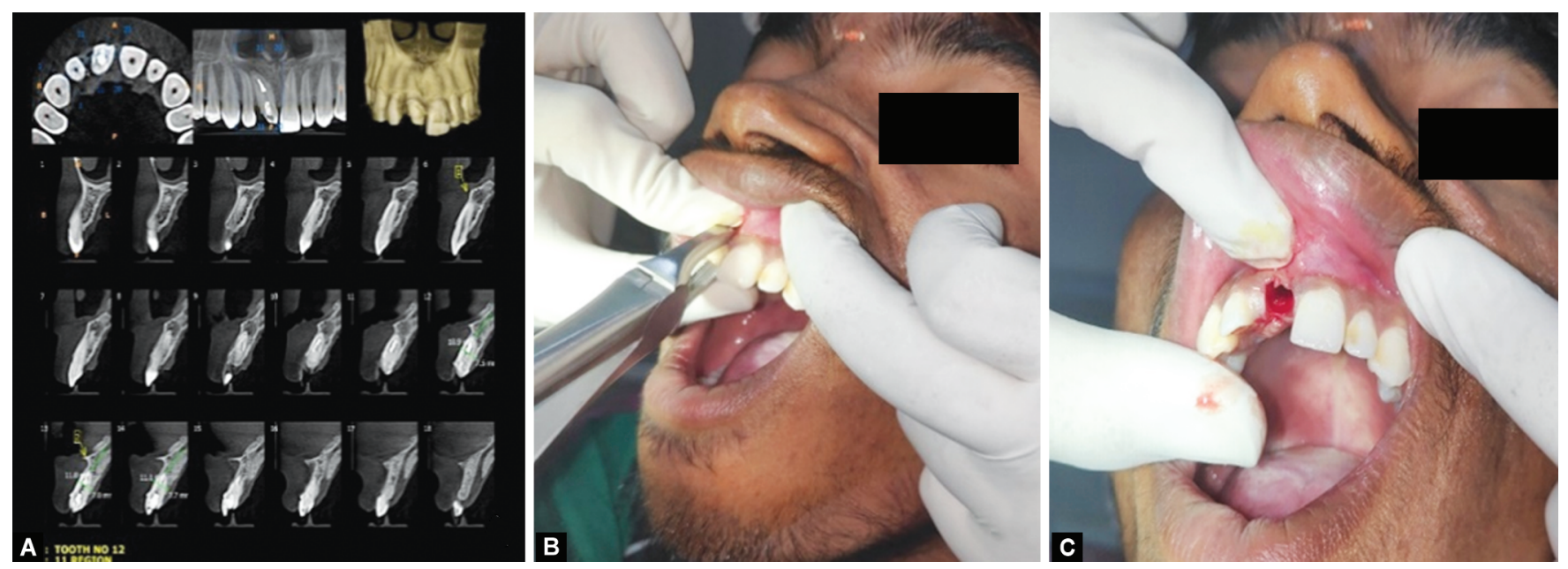

Figs 1 A to $C$ : (A) Preoperative $C B C T$; (B) Extraction of 11 ; (C) Extracted socket

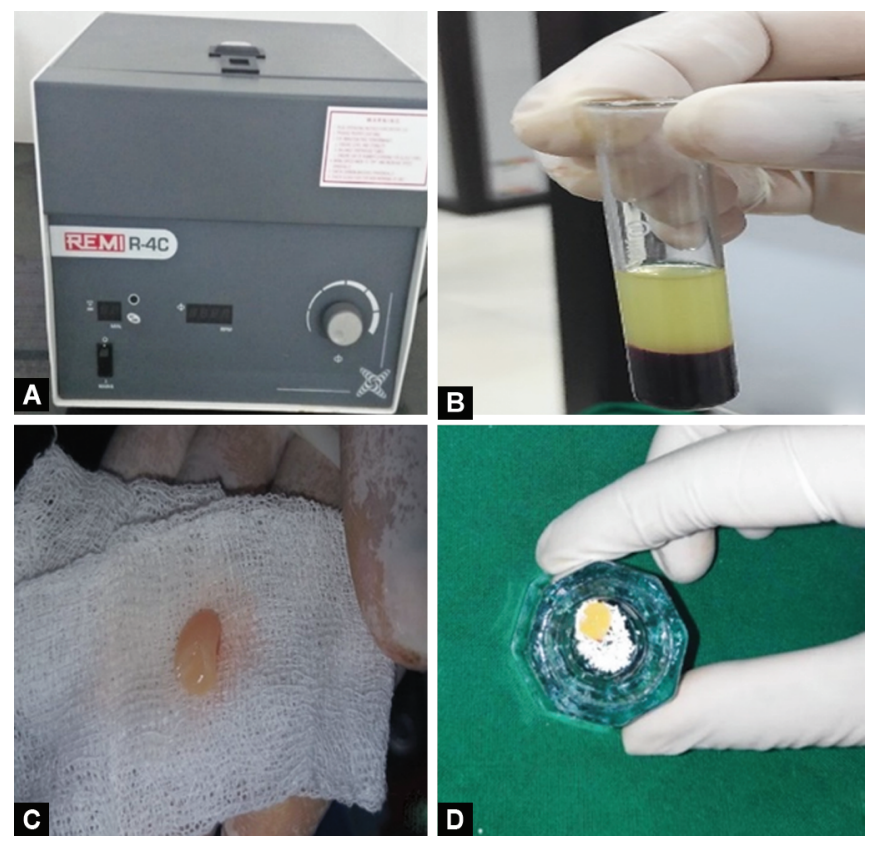

Figs 2A to D: (A) Platelet-rich fibrin (PRF) preparation in centrifuge; (B) Middle portion considered as PRF; (C) PRF; (D) Alloplast combination

influences the healing of hard and soft tissues as well as esthetics. ${ }^{5}$ There are several advantages that are associated with immediate placement, which include the following:

- Reduced treatment time.

- Maintenance of extraction socket.

- Less crestal bone loss.

- Increased patient satisfaction and treatment acceptance.

- This approach helps to preserve alveolar bone dimension, allowing placement of longer and wider implants and improving the crown-implant ratio. ${ }^{4}$

\section{Case Description}

A 22-year-old male patient reported to the Department of Prosthodontics and Crown and Bridge, Vinayaka Missions Sankarachariyar Dental College, Salem, Tamil Nadu, India. Patient's
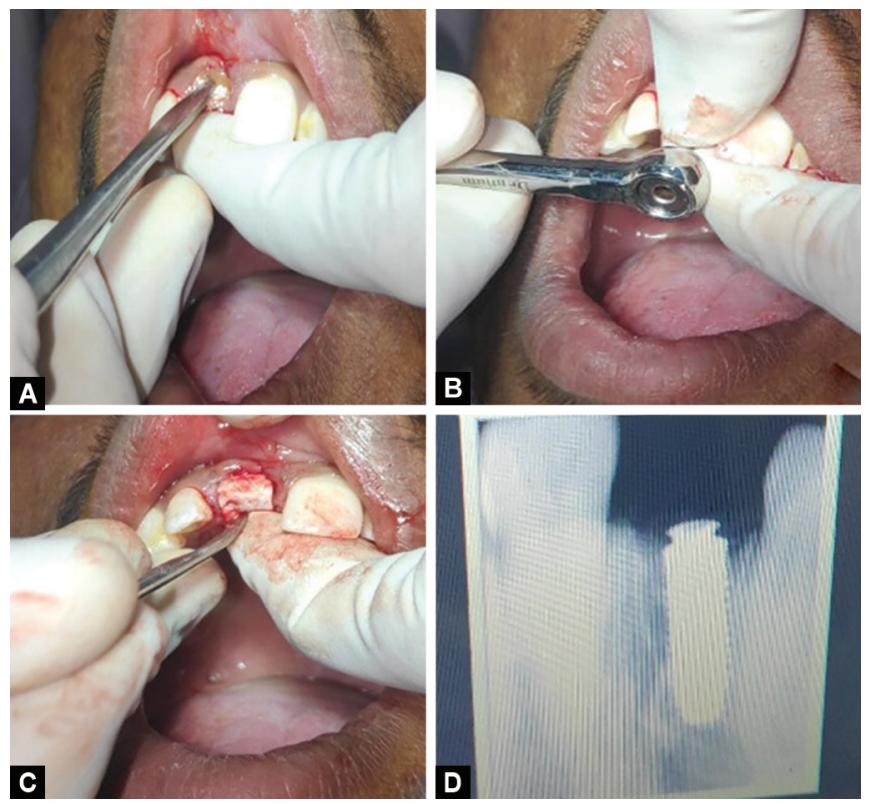

Figs 3 A to D: (A) Platelet-rich fibrin (PRF) and alloplast combination into the socket; (B) Implant placement was done; (C) GBR membrane was placed over the implant; (D) Postoperative RVG

chief complaint was frequent failure of crown in the right upper front tooth region (11), which was fractured and root canal treated. Hence, various replacement options like removable/fixed partial denture and immediate implant replacement were given. Further procedures were carried out with an informed consent.

Thorough intraoral and extraoral examination was done. Blood pressure and routine blood analysis were evaluated to assess the systemic health condition prior to treatment planning. A proper patient's history was taken and preoperative CBCT taken. Under the sterile condition, the procedure of immediate placement and extraction of the tooth was carried out by the surgeon and placement of the implant by the prosthodontist in an implant comprehensive clinical set-up (Figs 1 to 4).

Under local anesthesia, using anterior superior nerve block and nasopalatine nerve block, was given to the tooth need to be extracted. Using maxillary anterior tooth forceps, the right 

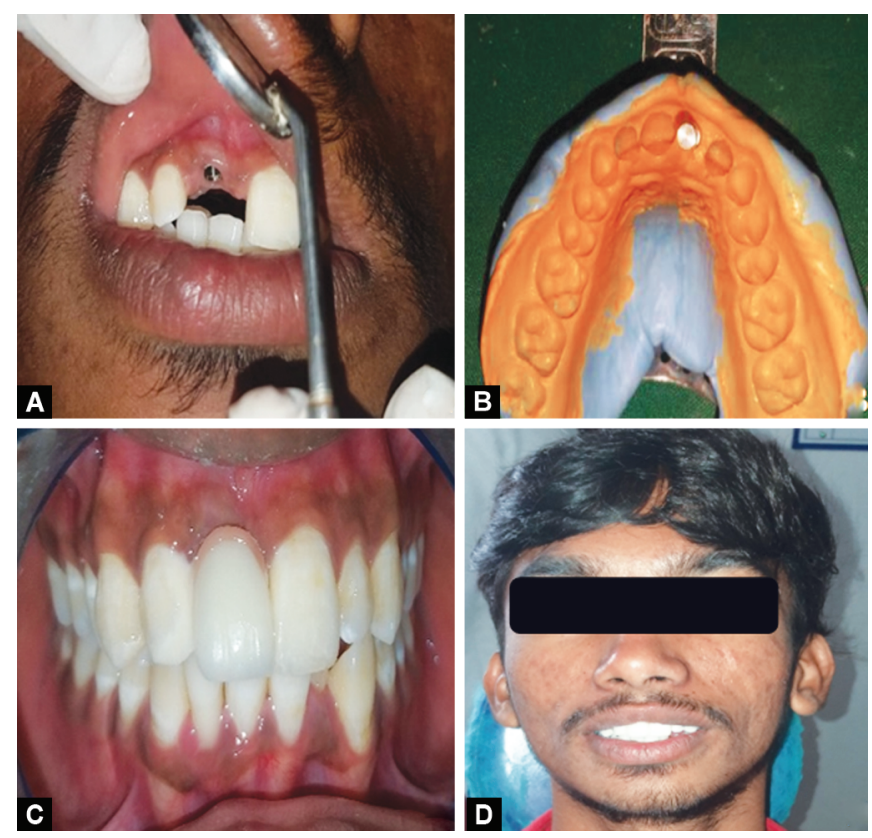

Figs 4A to D: (A) 6 months after removal of healing cap; (B) Implant-level impression; (C) Final metal ceramic restoration was given; (D) Implant prosthesis restored in patient

maxillary central incisor tooth was extracted. The extraction socket was evaluated for any remaining remnants and curettage was done. According to the extracted tooth length, the length of the implant was determined. After control of bleeding from the freshly extracted socket, initially lance drill of $2.0 \mathrm{~mm}$ was used; according to the diameter and length of the implant, osteotomy was carried out.

Minimal amount of osteotomy was carried out, which is less than the length of the implant planned for placement. The implant of required diameter and length $4.2 \times 13 \mathrm{~mm}$ was placed into the socket with proper torque to gain primary stability. Dentium Implant System was used in this present case.

Platelet-rich fibrin (PRF) preparation: It was obtained from the patient's venous blood $(5 \mathrm{~mL})$ collected and transferred into the test tubes without adding anticoagulant. Then, the test tube was kept in the centrifuge machine and centrifugation of blood was done at $3000 \mathrm{rpm}$ for 10 minutes. The resultant product consists of three layers, namely:

- Topmost layer consisting of a cellular plasma

- The PRF clot in the middle

- Red blood corpuscles base at the bottom

After this, it was necessary to put the PRF clot in a sterile petri dish for approximately 10 minutes to allow the release of the proper serum contained within, and it was placed onto the extracted socket along with the alloplast material, which is a combination of $60 \%$ hydroxy appetite and $40 \%$ tricalcium phosphate (B-OSTIN). These grafts were stabilized using the guided bone regeneration (GBR) membrane (Healiguide). The cover screw and sutures were placed and Coe-Pak was placed at the surgical site. Using betadine and saline, the implant-placed site was cleaned. Postoperative digital radiograph radiovisiography (RVG) was taken to evaluate the placement of the implant, Proper postoperative instructions were given; postoperative medications were prescribed to the patients. Oral hygiene instructions were given. Patients were recalled after
10 days to assess the implant site for wound healing and suture removal. After a week, the removable partial denture was provided. After 6 months of healing period and after the confirmation of osseointegration, intraoral periapical radiograph (IOPA) or RVG was taken. Then, second-stage surgery was carried out after 6 months, in which the cover screw replaced with the healing cap.

After 2 weeks of placement of the healing cap, the implant impression procedure was carried out. A stock tray was used to make a closed tray master impression. The healing cap was removed and the impression coping was inserted onto the superior portion of the implant surface.

The two-stage impression technique was carried out with the rubber-based impression material, then impression was taken. After disinfecting the impression, lab analogs were then connected to the impression copings and the master cast was poured with type IV dental stone. In impression, it is necessary to ensure proper fit. The jig trial was carried out on patients and fit was checked using radiographs (IOPA), and proper shade matching was done for better esthetics and appearance of the patient. Custom-made abutments were used and appropriate gingival shade was given to the implant crown and the fabricated crown was checked into the patient's mouth and zinc phosphate cement was used for implant crown cementation.

After setting of cement, excessive cements were removed. Using articulating paper, the patient was asked to do lateral and protrusive movements and premature contacts were reduced. Checked for proper occlusion. The implant maintenance instruction was given to the patient and subsequent follow-up was done after a week, 3 months, and 6 months and there was no evidence of failure, which shows a successful restoration.

\section{Discussion}

Platelet-rich fibrin was discovered by Choukroun et al. in 2001, which belongs to a new second generation of platelet concentrates. It releases growth factors and has lifetime between 7 days and 10 days. ${ }^{6}$ It is considered to be a wonderful tissue engineering product and shows promising results in wound healing and easier preparation. ${ }^{7}$ Alloplast is a synthetic graft material. It consist of $60 \%$ hydroxyapatite crystals and $40 \%$ of tricalcium phosphate. Various grafting materials have been used to fill the gap to ensure the constitution of the osseous interface with the implant. Beta tricalcium phosphate (b-TCP) is a biocompatible material that has been shown to influence osteoconduction, simultaneously with osteoblastic activity. ${ }^{8}$

Guided bone regeneration is performed simultaneously with other graft materials and is indicated only in narrow ridge defects and deficient bone height and width. This treatment concept advocates the regeneration of osseous defects, which mechanically exclude nonosteogenic cell structures, thereby allowing osteogenic cell populations originating from the parent bone to inhabit the osseous wound. ${ }^{9}$

The ideal goal of modern dentistry is to restore the patient to natural contour, function, comfort, esthetics, and health for the lifetime of that individual. The uniqueness of implants is that they can achieve this goal regardless of the condition of the stomatognathic system. An implant-retained prosthesis is almost independent of the remaining hard and soft tissues in the oral cavity for its support, thereby making it harmonious with the surrounding natural structures. The growing acceptance and popularity of this discipline can be justified by various functional significant reasons. 
In 1989, Lazzara first placed immediate implant placement at an extraction socket. ${ }^{10}$

Schwartz-Arad concluded after reviewing the relevant literature on immediate dental implants that the implants placed into fresh extraction sockets have a high rate of survival ranging between 93.9 and $100 \%$. ${ }^{1}$ Hafez conducted a study to evaluate the efficacy of PRF as a membrane for coverage of immediate implants in the maxillary anterior region. After 6 months, the marginal bone was stable in $83 \%$ of cases. Clinically, good soft tissue coverage was obtained. Radiographically, bone height showed a statistically significant decrease in the distal side while there was no statistically significant decrease at the mesial side. The change in defect depth was limited to $2 \mathrm{~mm}^{11}$

Schropp et al. concluded that new bone formation occurs associated with immediately placed implants in extraction sockets, which was similar to our results. ${ }^{12}$ An immediate implantation involves some risks, such as the higher likelihood of infection, flap dehiscence, and the occurrence of buccal bone remodeling that can lead to long-term marginal mucosa recession. ${ }^{13}$ But in this study, there was no signs of infection and flap dehiscence.

Schwartz-Arad, ${ }^{1}$ Schropp, ${ }^{12}$ and Younis ${ }^{14}$ conducted a study and concluded that immediate implant placement was found to have superior results over delayed implant placement.

In this case, immediate implant placement has greatly reduced the treatment time, shows excellent soft tissue healing, and promotes bone regeneration as a combination of graft materials like PRF, alloplast, and GBR was used. Platelet-rich fibrin is a readily available, cost-effective material that can be used for soft and hard tissue regeneration. Especially with vertical bone defect cases and esthetic areas, use of such combined graft materials will provide long-term success of the restoration.

\section{Conclusion}

Summing up, implants aid in better mastication and phonetics; are more comfortable for patients by reducing the size of the prosthesis; provide stability, retention, support, etc., almost similar to that of the natural dentition; avoid abutment teeth preparation; give a provision for combination (fixed-removable) prosthesis; improve the health of the adjacent structures; have a more predictable course of treatment; and by all these they ultimately improve the lifespan of the prosthesis in the patient's mouth. Platelet-rich fibrin shows excellent results as it was prepared from patient's autologous blood, which was rich in growth factor that promotes healing and as well as bone regeneration. It is cost-friendly, easily available, and has less preparation time.

Well-formed bone is seen in immediate implant placement. Hence, provided the patient's general health and economic conditions permit selecting it, the implant-retained prosthetic device will be a much better choice in most of the cases for the productive treatment in the longer run. ${ }^{3}$

\section{Clinical Significance}

The single tooth implant revealed a higher success rate, excellent wound healing, and soft tissue regeneration was found with the use of PRF and alloplast combination. Bone regeneration was well appreciated. Immediate implant placement with delayed loading had found to be a good alternative with a high success rate when compared to the conventional procedure.

\section{Detalls of Products Used}

- Manufacturer's name: Dentium Implant System, Seoul, Korea, and the United States

- Alloplast material: B-ostin, Chandigarh, India

- GBR: Healiguide, Chennai, Patented American Technology

\section{Patient Consent}

The patient has given his consent for his images and clinical information. The procedure was carried out with a proper consent from the patient by explaining the treatment.

\section{References}

1. Schwartz-Arad D, Chaushu G. Immediate implant placement: a procedure without incisions. J Periodontol 1998;69(7):743-750. DOI: 10.1902/jop.1998.69.7.743.

2. Mishra S, Chowdhary R, Patil P, et al. Replacement of missing central incisor with single implant-supported fixed prosthesis. J Curr Res Sci Med 2016;2(2):116. DOI: 10.4103/2455-3069.198383.

3. Sehgal M, Puri L, Yadav S. Immediate dental implants enriched with L-PRF in the esthetic zone. Case Rep Dent 2018;2018:1-6. DOI: $10.1155 / 2018 / 9867402$.

4. Bali A, Jindal M, Goel A, et al. Comparative evaluation of clinical and radiographical outcomes of immediate versus delayed dental implant placement: a prospective study. Indian J Dent Sci 2019;11(3):133. DOI: 10.4103/IJDS.IJDS_18_18.

5. Juodzbalys G, Wang H-L. Soft and hard tissue assessment of immediate implant placement: a case series. Clin Oral Implants Res 2007;18(2):237-243. DOI: 10.1111/j.1600-0501.2006.01312.x.

6. Borie E. Platelet-rich fibrin application in dentistry: a literature review. Int J Clin Exp Med 2015;8(5):7922-7929.

7. Bashir B. Platelet rich fibrin (PRF) in the surgical management of periapical lesions: a research article. Int J Appl Dent Sci 2019;5(2): 230-232.

8. Harel N, Moses O, Palti A, et al. Long-term results of implants immediately placed into extraction sockets grafted with $\beta$-tricalcium phosphate: a retrospective study. J Oral Maxillofac Surg 2013;71(2):e63-e68. DOI: 10.1016/j.joms.2012.09.022.

9. Ahmad M, Naim H, Balakrishnan D, et al. Implant placement with guided bone regeneration in the maxillary aesthetic zone - a case report with 3 years of follow up. Dent Oral Craniofacial Res [Internet] 2017(3). DOI: 10.15761/DOCR.1000206.

10. Tadi D, Vadapalli H, Gujjalapudi M, et al. Evaluation of initial stability and crestal bone loss in immediate implant placement: an in vivo study. J Int Soc Prev Community Dent 2014;4(3):139. DOI: 10.4103/2231-0762.142002.

11. Hafez WK, Seif SA, Shawky H, et al. Platelet rich fibrin as a membrane for coverage of immediate implants: case-series study on eight patients. Tanta Dent J 2015;12(3):203-210. DOI: 10.1016/ j.tdj.2015.05.009.

12. Schropp L, Wenzel A. Timing of single implant placement and long-term observation of marginal bone levels. Eur J Oral Implant 2016;9(Suppl 1):S107-S122.

13. Barbisan A. Soft tissues changes after immediate and delayed single implant placement in esthetic area: a systematic review,. J Oral Implantol 2015;41(5):612-619. DOI: 10.1563/AAID-JOI-D-13-00095.

14. Younis L, Taher A, Abu-Hassan MI, et al. Evaluation of bone healing following immediate and delayed dental implant placement. J Contemp Dent Pract 2009;10(4):35-42. DOI: 10.5005/jcdp-10-4-35. 